\title{
Online Optimization Voltage Control Based on Simplified Sensitivity Algorithm for Active Distribution Network
}

\author{
ZHUANG Huimin, ZHANG Jianglin \\ School of Control Engineering Chengdu University of Information Technology, \\ Chengdu 610225, Sichuan Province, China \\ ahuimin@cuit.edu.cn, ${ }^{b} 542228752 @ q q . c o m$
}

\begin{abstract}
Keywords: active distribution network; voltage online control; simplified sensitivity calculation; direct power flow algorithm; optimization model

Abstract. A real time control strategy based on sensitivity analysis is proposed for voltage regulation in active distribution network with perfect measurement and communication infrastructure. Based on distribution network direct power flow algorithm, a simplified calculation method of voltage sensitivity is presented for the real time control. This algorithm is simple and suitable for online control due to less calculation. The optimization model for voltage online control is made and resolved using standard linear programming algorithm. Then the optimization resolutions and the voltage measured by RTU were together applied for controlling the reactive resources to regulate the voltage. Numerical simulation results on the typical two-feeder distribution test system have showed that the proposed voltage regulation method is able to coordinate the controllable reactive power equipment to dynamically keep the voltage in its acceptable range.
\end{abstract}

\section{Introduction}

Driven by environmental, economic and technical incentives, an increasing number of distributed generation (DG) units have been integrated into distribution network. Proliferation of DGs has also brought about the concept of active distribution network (ADN), where variables are measured and controlled by means of various devices based on a centralized and intelligent system. However, the rapid penetration of DGs, especially renewable energy sources (RES) such as photovoltaic (PV) and wind generators, has brought new operation problems in ADN. Temporary over/under voltages and congested lines are part of the negative effects caused by intermittent production of RES [1]. This has given a great challenge for voltage regulation in ADN. As consequence, voltage control technology in $\mathrm{ADN}$ has been a heated topic in recent years.

Compared with the traditional distribution network (TDN), SCADA system and communication system are more advanced and the distribution of remote terminal units (RTU) is more extensive in ADN, which solves the problem of poor observability in TDN and also provides a new idea for voltage control in ADN. At present, some researchers improved inverter control strategies to reduce the impact of intermittent DG on distribution network [2-3], and others applied centralized off-line strategy for voltage control in active distribution network. In [4-5 ], an optimization algorithm for a day ahead scheduling is used to control voltage. In [6], artificial intelligence techniques have been developed to reduce the calculation time of solving optimal model. Although these control strategies achieve good performances, they require forecasted daily load and power output of RES, and their computing time is much, which does not let them to be used in real time schedule. An on-line optimal control approach based on the voltage sensitivity is presented in [7]. But the sensitivity calculation method is complicated and takes much time, so it needs to be further improved to meet the requirements of online control.

In this paper, a new approach is proposed for control and coordination programmable reactive power sources in ADN. The proposed method is performed based on the RTU data which are collected from the selected points of distribution feeders. These RTUs are installed on the nodes of programmable reactive power sources (RPS). Besides, a simplified voltage sensitively method based on the direct power flow algorithm is put forth, which is suitable for on-line calculation. 


\section{Simplified Voltage Sensitivity Algorithm}

It is a usual method for voltage control to use Jacobi matrix in Newton power flow algorithm to calculate voltage sensibility to injected reactive power [8]. However, it is of large calculation and so not suitable for online voltage control. In this paper, a simplified sensibility approach is proposed, which is based on the direct power flow (DLF). Here a simple 5-bus radial distribution system as an example is used to demonstrate the method principle.

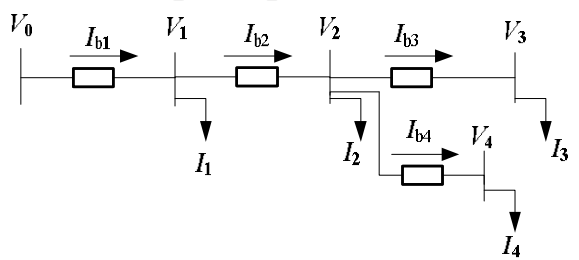

fig.1 A simple 5-bus radial distribution system

The branch current are obtained by applying the Kirchhoff's current law to the system in Fig1:

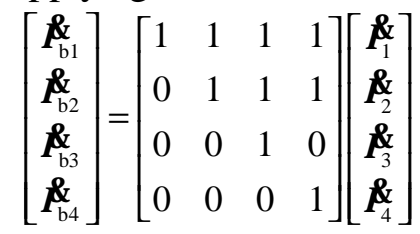

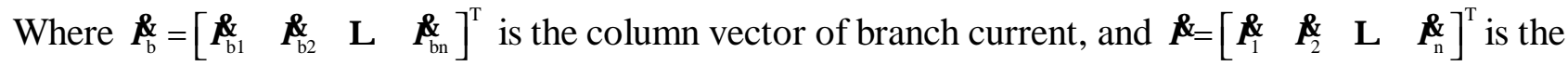
one of bus current injection at the system buses.

In general, the phase difference between the node voltage is small in distribution network, so the horizontal component of branch voltage drop can be omitted. The voltage drop between the node- $i$ and the node- $j$ is calculated approximately according to the formula (2):

$$
V_{i}-V_{j}=\frac{R_{i j} P_{i j}+X_{i j} Q_{i j}}{V_{j}}
$$

Where $R_{i j}, X_{i j}, P_{i j}, Q_{i j}$ are resistance, reactance, active power and reactive power of the branch-ij.

The formula (2) is applied in Fig1:

$$
\left[\begin{array}{l}
V_{0} \\
V_{0} \\
V_{0} \\
V_{0}
\end{array}\right]-\left[\begin{array}{l}
V_{1} \\
V_{2} \\
V_{3} \\
V_{4}
\end{array}\right]=\boldsymbol{V}^{\prime}\left[\begin{array}{cccc}
r_{01} & 0 & 0 & 0 \\
r_{01} & r_{12} & 0 & 0 \\
r_{01} & r_{12} & r_{23} & 0 \\
r_{01} & r_{12} & 0 & r_{24}
\end{array}\right]\left[\begin{array}{l}
P_{01} \\
P_{12} \\
P_{23} \\
P_{24}
\end{array}\right]+\boldsymbol{V}^{\prime}\left[\begin{array}{cccc}
x_{01} & 0 & 0 & 0 \\
x_{01} & x_{12} & 0 & 0 \\
x_{01} & x_{12} & x_{23} & 0 \\
x_{01} & x_{12} & 0 & x_{24}
\end{array}\right]\left[\begin{array}{l}
Q_{01} \\
Q_{12} \\
Q_{23} \\
Q_{24}
\end{array}\right]
$$

Where $V^{\prime}$ is a column vector with the element of $1 / V_{i}$ 。

Compared with the node power injection, branch power loss is much smaller so that it can be omitted. Therefore the follow formula is derived from the formula (3):

$$
\boldsymbol{V}_{0}-\boldsymbol{V}=\boldsymbol{V}^{\prime}\left[\begin{array}{cccc}
r_{01} & r_{01} & r_{01} & r_{01} \\
r_{01} & r_{1}+r_{12} & r_{01}+r_{12} & r_{01}+r_{12} \\
r_{01} & r_{01}+r_{12} & r_{01}+r_{12}+r_{23} & r_{01}+r_{12} \\
r_{01} & r_{01}+r_{12} & r_{01}+r_{12} & r_{01}+r_{12}+r_{24}
\end{array}\right]\left[\begin{array}{c}
P_{1} \\
P_{2} \\
P_{3} \\
P_{4}
\end{array}\right]+\boldsymbol{V}^{\prime}\left[\begin{array}{cccc}
x_{01} & x_{01} & x_{01} & x_{01} \\
x_{01} & x_{01}+x_{12} & x_{01}+x_{12} & x_{01}+x_{12} \\
x_{01} & x_{01}+x_{12} & x_{01}+x_{12}+x_{23} & x_{01}+x_{12} \\
x_{01} & x_{01}+x_{12} & x_{01}+x_{12} & x_{01}+x_{12}+x_{24}
\end{array}\right]\left[\begin{array}{l}
Q_{1} \\
Q_{2} \\
Q_{3} \\
Q_{4}
\end{array}\right]
$$

The formula (4) is extended to the distribution network with $N$ nodes, another formula is as follow:

$$
\Delta \boldsymbol{U}=\boldsymbol{V}(\boldsymbol{R P}+\boldsymbol{Q X})=\boldsymbol{R}_{\mathrm{v}} \boldsymbol{P}+\boldsymbol{Q}_{\mathrm{v}} \boldsymbol{X}
$$

Where diagonal element $R_{i i}$ (or $X_{i i}$ ) of resistance (or reactance) matrix is called the self resistance (or reactance), which is defined as the sum of lines' resistance (or reactance) lacated between substation and injected power bus; other elements $R_{i j}$ (or $\left.X_{i j}\right)(i \neq j)$ are called the mutual resistance (or reactance) and are defined as the sum of lines' resistance (or reactance) which is common between substation bus and bus- $i$ and bus- $j . R_{\mathrm{V}}$ and $X_{\mathrm{V}}$ are $n$-order square matrixed with the elements of $R_{i j} / V_{j}$ and $X_{i j} / V_{j}$ respectively.

$V_{0}$ is supposed to keep constant, node- $i$ voltage sensibility to node- $j$ injected power is derived as follow from the formula (5): 


$$
S_{\mathrm{p} i j}=\frac{\partial V_{i}}{\partial P_{j}}=-\frac{R_{i j}}{V_{j}}, S_{\mathrm{q} i j}=\frac{\partial V_{i}}{\partial Q_{j}}=-\frac{X_{i j}}{V_{j}}
$$

The formula(6) shows that the voltage sensitivity coefficients are obtained from DLF matrix in the load flow study. The real part of DLF matrix in each array composes the matrix R and its imaginary part gives the matrix $\mathrm{X}$. The sensibility is mainly dependent on topology of distribution network.

According to the formula (6), volgage variation caused by reactive change can be calculated as follow:

$$
\Delta \boldsymbol{V}_{n \times 1}=\boldsymbol{X}_{V n \times m} \times \Delta \boldsymbol{Q}_{m \times 1}
$$

Where $\Delta V$ is a $n$-dimensional column vector with the element of node voltage variation, and $\Delta Q$ is a $m$ - dimensional column vector with the element of node injected reactive variation.

\section{Online Voltage Control Strategy}

(1) Online Voltage Control System Structure

There are more advanced SCADA system and communication network in ADN compared with TDN. And the measured data are more accurate and remote control is more reliable. It is make it more easy to control reactive power and voltage based on SCADA system. So the proposed system structure in this paper is shown in Fig.2

In this system, one RTU was installed on each controllalbe reactive power source's bus. Each RTU should measure local bus voltage and then dilver it to the master SCADA. These received data from RTUs are fed to Distribution Management System (DMS). The online optimization program can be started to run as soon as voltages out of limits are monitored. After that, the master SCADA sends a commond to adjust RPS through a communication link. Finally, the RPS controller receives an input from the designated RTU and adjust its reactive power output accordingly. .

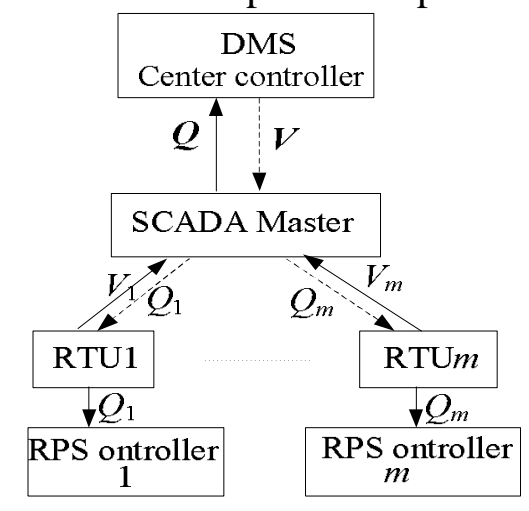

Fig.2 voltage control system structure

(2) Online Voltage Optimal Control Model

Since the injected reactive power may be positive or negative, the injected reactive power increment on node- $j$ is decomposed into the form of (8) so as to conform to the form of the standard linear programming

$$
Q_{j}(k+1)=Q_{j \mathrm{p}}(k+1)-Q_{j \mathrm{n}}(k+1)
$$

Where $Q_{j}(k+1) 、 Q_{j \mathrm{p}}(k+1) 、 Q_{j \mathrm{n}}(k+1)$ are the injected reactive power increment on node- $j$ at $k+1$ moment and its positive part and negtive one respectively. $Q_{j \mathrm{p}}(k+1)$ and $Q_{j \mathrm{n}}(k+1)$ are both positive.

If $Q_{j}(k+1)>0$, let $Q_{j \mathrm{n}}(k+1)=0$, If $Q_{j}(k+1)<0$, let $Q_{j \mathrm{p}}(k+1)=0$.

The voltage on the monitored node at $k+1$ moment is :

Objective:

$$
V(k+1)=V(k)+X_{\mathrm{V}}(k)\left(Q_{\mathrm{p}}(k+1)-Q_{\mathrm{n}}(k+1)-Q(k)\right.
$$

$$
\min \sum_{j=1}^{m}\left(Q_{j \mathrm{p}}(k+1)+Q_{j \mathrm{n}}(k+1)\right)
$$

Constraints: 


$$
\begin{gathered}
V_{\min } \leq V(k+1) \leq V_{\max } \\
0 \leq Q_{j \mathrm{p}}(k+1) \leq Q_{j \max }, 0 \leq Q_{j n}(k+1) \leq Q_{j \max }
\end{gathered}
$$

The linear programming method suitable for online optimisation is used to resolve the optimal model composed of (8) to (11), and obtain setting values of RPS.

\section{Simulation}

Fig. 3 shows a single line diagram of a typical distribution network consisting of two feeders. The two feeders are supplied by two 20MVA, $110 / 10 \mathrm{kV}$. Voltage limits are taken to $\pm 5 \%$ of nominal. The impedance of each branch is $0.79+\mathrm{j} 0.73 \Omega$. The unit reactive power capacity of each capacitor bank (expressed by ' $q$ ') is $150 \mathrm{kvar}$, the maximum step of banks 1 and 2 is 6 , and one of banks 3 and 4 is 8 . Two wind turbines both operate in reactive power control model with $Q_{\max }=450$ kvar.

The main bus voltage is fixed at 1 pu by transformer tap changer. The measured data by RTU are from the direct power flow algorithm. The active power and capacitive reactive power injected by DG are both negative and inductive reactive power is positive. The output power of wind turbines is shown in Fig.4. The load consumption at each low voltage feeders as well as their parameters are referenced the paper [6].

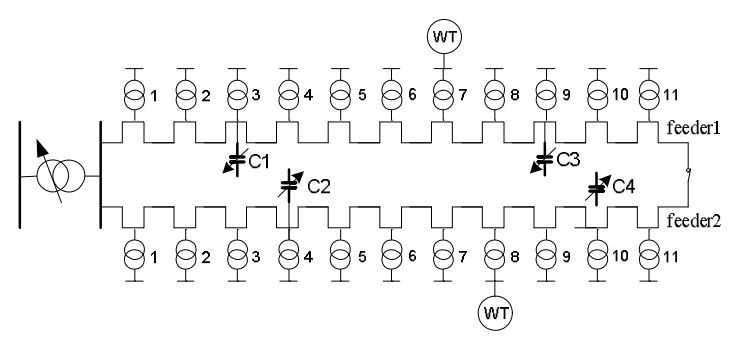

Fig. 3 Two-feeder distribution test system

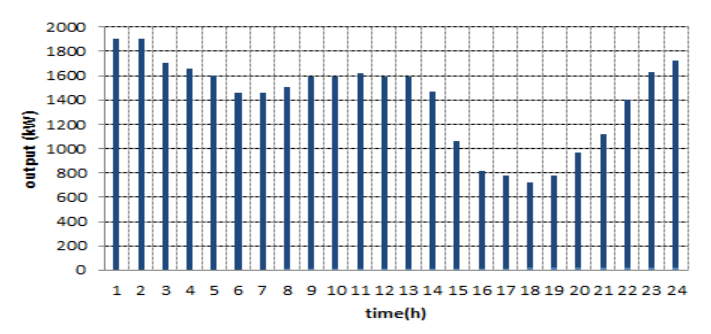

Fig. 4 Wind power generation

In this paper, modeling and simulation were executed based on Matlab / Simulink. The simulation results are shown in Figure 5-10. The results of switched capacitors steps arrangement are shown in the Figs.5-6. And the output reactive power of wind turbines is shown in Fig.7. As seen from Fig.5-7, the steps of the capacitor banks and the reactive power output of the wind turbine reduce at off-peak hours, and on the other hand, they increase at the peak hours.

Voltage of bus 11 in both feeders during 24 hours is shown in Fig. 8. It can be seen that the voltage has been kept within the range of 0.96 to 1.03 all the time. Fig. 9 shows the voltage of each bus node when the load is light and the wind power is large. Fig. 10 shows the voltage under heavy load. From these two figures, it can be seen that the voltage of each node has been always kept in the allowable voltage range, whether under light load or heavy load.

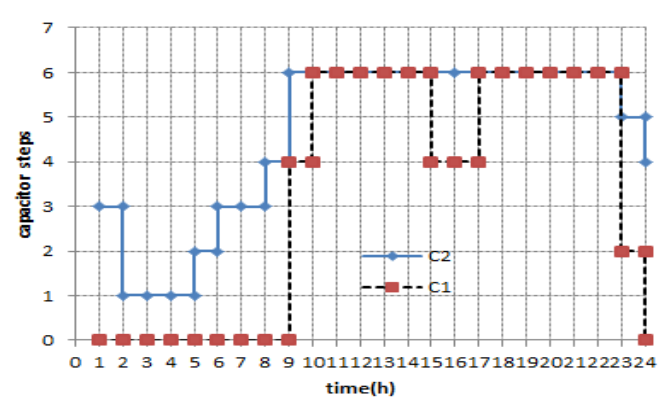

Fig. 5 Steps of capacitors 1 and 2

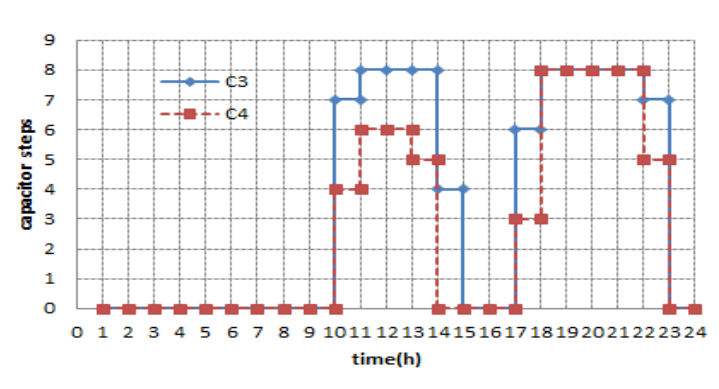

Fig. 6 Steps of capacitors 3 and 4 


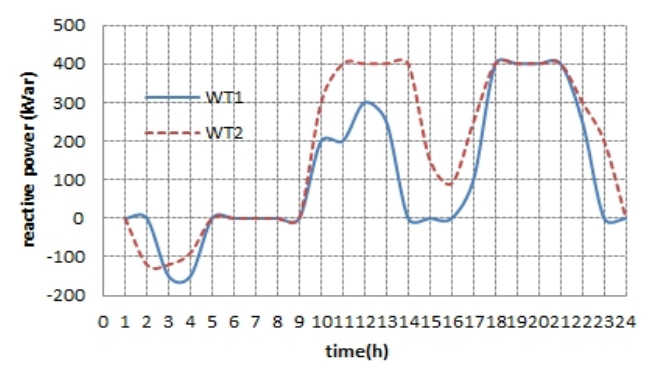

Fig. 7 Reactive output of wind power generator

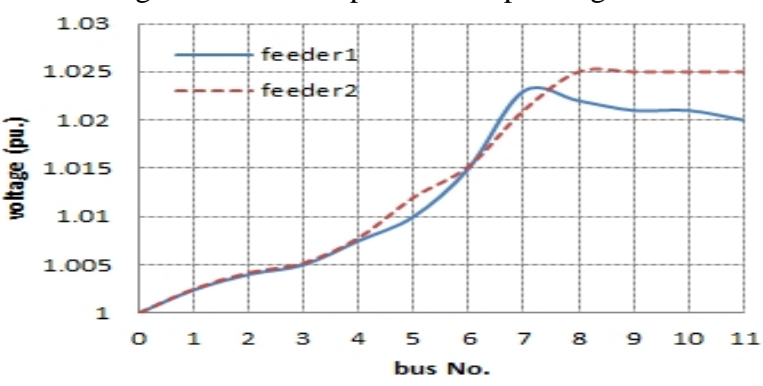

Fig. 9 Voltage of feeders 1 and 2 at 3 a.m.

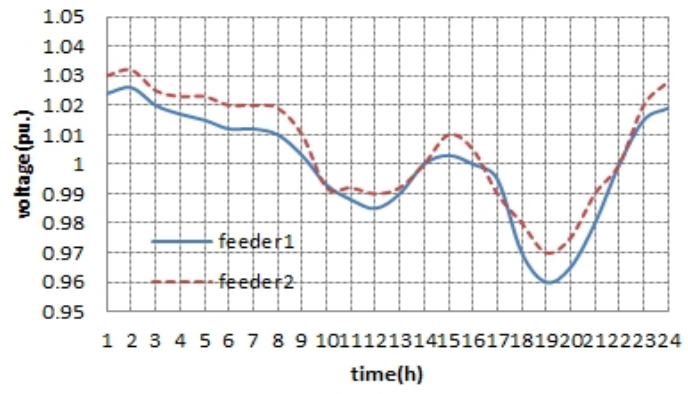

Fig. 8 Voltage profile of bus 11 on two feeders

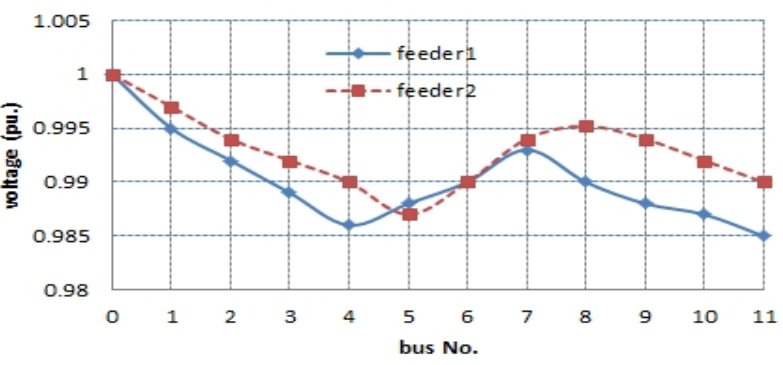

Fig. 10 Voltage of feeders 1 and 2 at 13 a.m.

\section{Conclusion}

This paper proposed a simplified sensitivity algorithm based on direct power flow algorithm according to the characteristics of ADN, and a voltage optimal control model is established to execute on-line optimal control. The technique is based on locating RTU at each controllable RPS' buses. Unlike previous voltage control methods, the proposed approach needn't the load and renewable generations forecast data, and are carried out online. In addition, the simulation results show that the proposed method can keep the voltage in its predefined range whenever under light load or heavy load. So the proposed control strategy is efficient and could be integrated into present control system.

\section{References}

[1] Walling R A, Saint R, Dugan R C, et al. Summary of distributed resources impact on power delivery systems [J] . IEEE Trans. On Power Delivery, 2008, 23(3), pp.1636-1644.

[2] Fan Mingtian, Zhang Zuping, Su Aoxue, et al. Enabling technologies for active distribution systems [J]. Power System Protection and Control, 2015, 43(15), pp.67-72.

[3] Mahdad B, Srairi K. Solving multi-objective optimal power flow problem considering wind-STATCOM using dirential evolution. IEEE Trans power Del 2013, 27(2): 75-89

[4] Kashem M., Michael N., Gerard Ledwich. A Coordinated Voltage Control Approach for Coordination of OLTC, Voltage Regulator, and DG to Regulate Voltage in a Distribution Feeder [J]. IEEE Transaction on Industry Applications, 2015, 51(2), pp.1239-1248.

[5] ZHUANG Huimin, XIAO Jian. A Two-Stage Optimal Schedule Model for the Active Distribution Network [J] . Journal of Southwest Jiaotong University, 2015, 50(05), pp.928-934

[6] XING Haijun, CHENG Haozhong, ZHANG Yi. Reactive Power Comprehensive Optimization in Distribution Network Based on Multiple Active Management Schemes [J] . Power System Technology, 2015, 39(6): 1504-1510 
[7] K.Christakou, J.LeBoudec, M.Paolone. Efficient computation of sensitivity coefficients of node voltage and line currents in unbalanced radial electrical distribution networks [J]. IEEE Trans. Smart Grid, 2013, 4(2): 741-750

[8] Teng Jen-Hao. A direct approach for distribution system load flow solutions[J]. IEEE Trans Power Delivery, 2003, 18(3):882-7 